\title{
Improving the Quality of EEG Data in Patients With Alzheimers Disease Using ICA
}

\author{
François-Benoit Vialatte $^{1}$, Jordi Solé-Casals ${ }^{2}$, Monique Maurice ${ }^{1}$, Charles \\ Latchoumane $^{3}$, Nigel Hudson ${ }^{4}$, Sunil Wimalaratna ${ }^{5}$, Jaeseung Jeong ${ }^{3}$, and \\ Andrzej Cichocki ${ }^{1}$ \\ 1 Riken BSI, Lab. ABSP, Wako-Shi, Japan, \\ fvialatte@brain.riken.jp \\ 2 University of Vic, Signal Processing Group, Vic, Spain \\ 3 KAIST, Dept of Bio and Brain Engineering, Daejeon, South Korea \\ 4 Derriford Hospital, Dept of Neurophysiology, Plymouth, UK \\ ${ }^{5}$ Radcliffe Infirmary, Dept of Neurology, Oxford, UK
}

\begin{abstract}
Does Independent Component Analysis (ICA) denature EEG signals? We applied ICA to two groups of subjects (mild Alzheimer patients and control subjects). The aim of this study was to examine whether or not the ICA method can reduce both group differences and within-subject variability. We found that ICA diminished Leave-OneOut root mean square error (RMSE) of validation (from 0.32 to 0.28 ), indicative of the reduction of group difference. More interestingly, ICA reduced the inter-subject variability within each group $(\sigma=2.54$ in the $\delta$ range before ICA, $\sigma=1.56$ after, Bartlett $\mathrm{p}=0.046$ after Bonferroni correction). Additionally, we present a method to limit the impact of human error ( $\simeq 13.8 \%$, with $75.6 \%$ inter-cleaner agreement) during ICA cleaning, and reduce human bias. These findings suggests the novel usefulness of ICA in clinical EEG in Alzheimer's disease for reduction of subject variability.
\end{abstract}

\section{Introduction}

Independent component analysis (ICA) is a method for recovering underlying signals from linear mixtures of those signals. Independent component analysis (ICA) is especially useful to reject EEG artifacts [1], exploiting statistical independent criteria to separate EEG sources, which allows to remove artifacts and clean EEG [2-4]. Therefore, it has been used for analysis of various physiological time series including the EEG. However, whether the ICA alters the EEG distributions or not is unclear. In addition to instrumental noise and environmental noise, movement and other physiological noise (ocular, electromyographic, electrodermal, electrovascular, and respiratory signals) may interfere with the EEG in the form of artifacts. Artifacts in the EEG can be defined as any potential difference due to an extra-cerebral source [5]. Particularly, muscle artifacts are especially problematic, because they can appear in EEG patterns which are very hard to differentiate from the EEG signals: the frequency range of muscle artifacts and the EEG overlap to a high degree [6]. 
The aim of this study was to address the significant question: is it a valid approach to improve EEG signals using Independent component analysis (ICA)? We investigated the effects of ICA cleaning on a database recorded from patients with Alzheimer's disease (mild AD, early stage) and healthy control subjects. It is of importance to investigate the possible application of ICA to AD, because the early diagnosis of AD using EEGs is very critical [7]. Previous studies used ICA to enhance differences between control subjects and Alzheimer patients, but the effect on EEG quality was only assessed by estimating the group separation (see e.g. $[3,8,9]$ - which is not sufficient to prove that ICA preserved EEG data. In this study, we used the ICA to show the following results:

1. Human performance displayed a high variability. We used a simple method to combine signals cleaned without concertizing by three scientist, which provided us with an optimally cleaned database (in respect to an Euclidean distance).

2. With proper precautions, ICA cleaning did not denature EEG signals.

Using the simple cleaning rules introduced in this study, it shall be possible to design a semi-automatic method to improve EEG quality.

\section{Methods}

Computations were done with Matalb (The MathWorks, Inc.), ICA cleaning was performed using ICALAB ver. 3 with automatic sorting of independent components [10].

\subsection{EEG Data - Patients With MildAD}

These data were obtained using a strict protocol from Derriford Hospital, Plymouth, U.K. and had been collected using normal hospital practices [11]. EEGs were recorded during a resting period with various states: awake, drowsy, alert and resting states with eyes closed and open. All recording sessions and experiments proceeded after obtaining the informed consent of the subjects or the caregivers and were approved by local institutional ethics committees. EEG dataset is composed of 24 healthy control subjects (age: $69.4+11.5$ years old; 10 males) and 17 patients with mild AD (age: $77.6+10.0$ years old; 9 males). The patient group underwent full battery of cognitive tests (Mini Mental State Examination, Rey Auditory Verbal Learning Test, Benton Visual Retention Test, and memory recall tests). The two groups are not perfectly age-matched, which might pose bias later on, but it was shown that no major effect was found due to this disparity [11]. The EEG time series were recorded using 19 electrodes disposed according to Maudsley system, similar to the 10-20 international system, at a sampling frequency of $128 \mathrm{~Hz}$. EEGs were band-pass filtered with digital 2nd order Butterworth filter (forward and reverse filtering) between 0.5 and 30 $\mathrm{Hz}$ (a sampling rate of $128 \mathrm{~Hz}$ means that frequencies above $25 \mathrm{~Hz}$ cannot be reliably studied [12]). 


\subsection{Independent Component Analysis}

Blind Source Separation (BSS) consists in recovering a set of unknown sources from their observed mixture $\mathbf{x}$. The linear and instantaneous models of BSS can be formulated as:

$$
\mathbf{x}=\mathbf{A s},
$$

where $\mathbf{s}$ represents a data matrix having as rows the observed signals, and $\mathbf{A}$ is the mixing matrix. According to the currently prevailing view of EEG signal processing, a signal can be modeled as a linear mixture of a finite number of brain sources, with additive noise(see e.g. [2-4]). Therefore, blind source separation techniques can be used advantageously for decomposing raw EEG data to brain signal subspace and noise subspace. If sources are supposed to be independent, then BSS can be called ICA.

The Second-Order Blind Identification (SOBI) algorithm is a well-known blind source separation (BSS) method for source signals with temporal structures and distinct spectra (AR processes). It already proved to be useful in many biomedical applications. A weight adjusted version of SOBI was suggested in [14]. SOBI jointly (approximately) diagonalizes time-delayed covariance matrices for many time delays. However, SOBI algorithm does not specify how many and which time delays to choose. An efficient weight adjusted variant of SOBI called IWASOBI $[13,15]$ was recently developped to solve this problem. The original weight adjusted SOBI used a standard AJD (Approximative Joint Diagonalization) algorithm.IWASOBI uses instead an AJD based on family of WEDGE ${ }^{1}$ algorithms [13]. For IWASOBI the number of jointly diagonalized covariance matrices can be relatively low in comparison to the standard SOBI while performance can be considerably higher. This algorithm allows reliable separation of $100+$ sources with temporal structure (autoregressive sources) in order of seconds. In our experiments we used the IWASOBI algorithm implemented in ICALAB ver.3 [10].

\subsection{Cleaning Rules}

Three EEG researchers visually inspected EEGs, and chose the least corrupted (artifact-clean) continuous $20 \mathrm{sec}$ interval of each recording for the analysis. Each trial was then decomposed using ICA. Sources were ordered using a kurtosis measure, and the researchers cleared up to seven sources per trial corresponding to artifacts (eye movements, EMG corruption, EKG, etc), using three criteria (see Fig.1):

1. Abnormal scalp distribution of the reconstructed channels (only a few electrodes contribute to the source, with an isolated topography)

2. Abnormal wave shape (drifts, eye blinks, sharp waves, etc.)

3. Source of abnormally high amplitude $(\geq 100 \mu \mathrm{V})$

\footnotetext{
${ }^{1}$ Weighted Exhaustive Diagonalization using Gauss itEration
} 
We have focused our attention mainly on the smallest and largest values of kurtosis (i.e. a measure of sparsity and distance to Gaussianity), which are more likely to be representative of artifacts. After this step, the remaining sources were back-projected onto the scalp, yielding an artifact clean data.

(a)

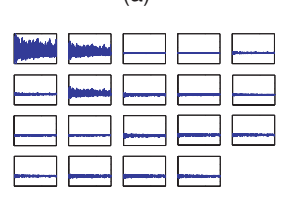

(b)

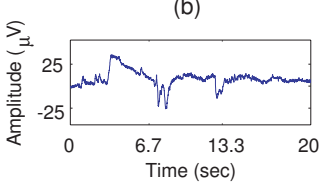

(c)

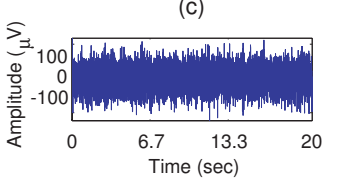

Fig. 1. Examples of artifacts. (a) abnormal scalp distribution of the reconstructed channels; (b) abnormal wave shape; (c) source of abnormally high amplitude.

\subsection{Optimal Combination of Three Cleanings}

Each of the three researchers cleaned, with the above rules, raw data from all subjects (EEG from mildAD patients or control subjects) $s_{n}$ with $n \in[1-$ 41]. This produced three cleaned databases: $\mathbf{D}_{\mathbf{1}}, \mathbf{D}_{\mathbf{2}}$ and $\mathbf{D}_{\mathbf{3}}$. Our objective was to combine efficiently these $\mathbf{D}_{\mathbf{i}}$ databases into one optimal database $\boldsymbol{\Omega}$. Each database $\mathbf{D}_{\mathbf{i}}$ includes cleaned $\mathrm{EEG}^{2} c_{i, n}$ with $n \in[1-41]$. We computed for all $c_{i, n}$ the averaged Fourier relative power $\boldsymbol{\Phi}(i, n, f)$ over all $E$ electrodes:

$$
\boldsymbol{\Phi}(i, n, f)=\frac{1}{E} \sum_{e=1}^{E} \boldsymbol{\Phi}_{i, n}(e, f) .
$$

Where $\boldsymbol{\Phi}_{i, n}(e, f)$ is the Fourier relative power of the electrode $e$ with frequencies $f$ in $\mathbf{F}=[1-25] \mathrm{Hz}$, for the cleaned EEG $c_{i, n}$. Fourier power was computed using the Welch method (1 sec. Hanning windows with $50 \%$ overlap). We then computed for each pair of cleaned EEG $\left(c_{i, n}, c_{j, n}\right)$ the Euclidian distance $\boldsymbol{\Delta}$ :

$$
\boldsymbol{\Delta}(n, i, j)=\sqrt{\sum_{f i n \mathbf{F}}\left[\boldsymbol{\Phi}\left(c_{i, n}, f\right)-\mathbf{\Phi}\left(c_{j, n}, f\right)\right]} .
$$

This distance is symmetric, with values in $\mathbb{R}^{+}$, and $c_{i, n} \simeq c_{j, n} \Rightarrow \boldsymbol{\Delta}(n, i, j) \simeq 0$. $\boldsymbol{\Delta}$ was used to evaluate significant differences between cleanings. To this end we used a Monte-Carlo approach to estimate the distribution of randomly matched surrogate data. The surrogate database was constituted of $r=10000$ draws of randomly matched data from the three cleaned databases. We computed the $r$

\footnotetext{
${ }^{2}$ corresponding to the raw data of $s_{n}$ : for each subject $s_{n}$ exist three cleaned EEG, $c_{1, n}, c_{2, n}$, and $c_{3, n}$
} 
distances $\boldsymbol{\Delta}(r, 1,2)$ of each pair, which allowed us to estimate the distribution of non-matched data. We fixed the significance threshold $\tau=1.1 \%$, as the fifth percentile of this distribution (if $\boldsymbol{\Delta}(n, i, j) \leq \tau$, we reject the hypothesis of having a significant difference between cleaners $i$ and $j$ for subject $s_{n}$ with a probability $\left.p=0.05^{3}\right)$.

For each of subject $s_{n}, n \in[1-41]$, we iteratively selected the best candidate $\omega_{n} \in \boldsymbol{\Omega}$ within the corresponding cleaned EEG triplet $\left[c_{1, n}, c_{2, n}, c_{3, n}\right]$ using the following decision rule:

1. Equivalent case: if $\forall\left(c_{i, n}, c_{j, n}\right), \boldsymbol{\Delta}(n, i, j)<\tau$, we select randomly the cleaned EEG $\omega_{n}$.

2. Consensus case: if $\forall\left(c_{i, n}, c_{j, n}\right), \exists$ only $\left(c_{u, n}, c_{v, n}\right), \boldsymbol{\Delta}(n, u, v) \geq \tau$ (or conversely $\boldsymbol{\Delta}(n, v, u) \geq \tau)$, we select the remaining cleaned EEG $\omega_{n}=c_{w, n}$ with $w \ni[u, v]\left(c_{w, n}\right.$ is a consensus of the two others cleaned EEG).

3. Error case: if $\exists u, \forall i, \boldsymbol{\Delta}(n, u, i) \geq \tau$, we select randomly $\omega_{n}=c_{v, n}$ with $v \neq u$ $\left(c_{u, n}\right.$ is identified as an error).

4. Reject case: if $\forall\left(c_{i, n}, c_{j, n}\right), \boldsymbol{\Delta}(n, i, j) \geq \tau$, the subject $s_{n}$ has to be rejected.

\section{Results}

\subsection{Variability Between Cleaners}

The cleaned data were distributed in the four above categories as follows: $75.6 \%$ equivalent cases, $17.1 \%$ consensus cases, $7.3 \%$ error cases and $0 \%$ reject cases $^{4}$. In other words, we could clean $100 \%$ of the database without rejection. From these numbers we can also obtain an approximate estimation of variability between human cleaners:

- Inter-cleaner agreement $\simeq 75.6 \%$

- Human error rate for one isolated cleaner $\simeq 13.8 \%$.

- Expected cleaning error $\%$ between two persons $\simeq 10.6 \%$.

This indicates that EEG data containing a significant number of subjects, cleaned by only one or two persons, is not so reliable.

\subsection{Group Differences}

Mann-Whitney z-scores were estimated before and after ICA cleaning. We observed, for all frequency ranges, that the differences between mildAD patients and control subjects were magnified by ICA (see Table 1 and Fig.2). We aggregated the Fourier power into five regions (frontal, temporal left and right, central and occipital). Linear discriminant analysis was applied before and after ICA cleaning. We estimated Leave-One-Out root mean square error (RMSE) of validation: it dropped from 0.32 to 0.28 (training RMSE dropped from 0.30 to 0.26). This shows that the topography of EEG relative powers after ICA cleaning was more consistent than those before cleaning.

\footnotetext{
$\overline{{ }^{3} \text { A probability }} p=0.01$ corresponds to a threshold $\tau=0.89 \%$

${ }^{4}$ This result is off course dependant on the threshold $\tau$.
} 
Table 1. Mann-Whitney Zscore before and after ICA, for each frequency range (comparing mildAD patients vs. control subjects). Higher absolute value of z-score means that p-value is lower (i.e. that data is better separated).

\begin{tabular}{|c|c|c|c|c|}
\hline Data & $\delta(1-4 \mathrm{~Hz})$ & $\theta(4-8 \mathrm{~Hz})$ & $\alpha(8-12 \mathrm{~Hz})$ & $\beta(12-25 \mathrm{~Hz})$ \\
\hline Before ICA & 3.68 & 2.83 & -4.39 & -3.78 \\
\hline After ICA & 3.76 & 4.82 & -4.58 & -4.42 \\
\hline
\end{tabular}

\subsection{Inter-Subject Variability}

After ICA cleaning, we examine if EEG was not denatured. This can be observed through the distribution of Fourier power in the two groups: did the subjects of control and patient group resembled each-other more after ICA, or instead did they differ more (in this case, EEG is denatured).

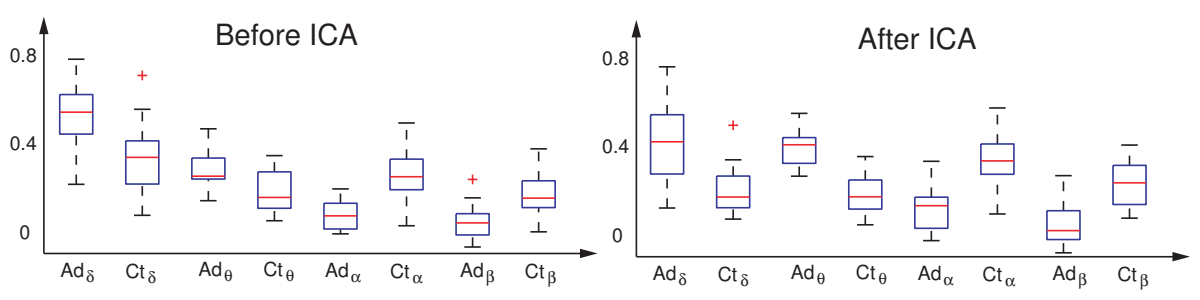

Fig. 2. Boxplot of Fourier relative power before and after ICA. Central line represents the median, dashed lines covers inter-quartile range, cross are outliers. ICA magnified the group differences.

We evaluated the inter-subject variability in both groups, by comparing their variance using a Bartlett test for homoscedasticity (before vs. after ICA). Variance was never shown to increase (even without Bonferroni correction), instead a significant decrease was found in the $\delta$ range for the control group (standard deviation $\sigma=2.54$ before ICA, $\sigma=1.56$ after, Bartlett $\mathrm{p}=0.046$ after Bonferroni correction). This decrease in inter-subject variability is also found generally: ICA subjects became closer to their group's mass centers (see Fig.3).

\section{Discussion and Conclusion}

We found that ICA cleaning improved the separation between mildAD patients and control subjects (confirming previous studies $[4,8,9]$ ). Moreover, we found a more consistent topographical distribution of EEG power after ICA. We could conjecture two possibilities concerning the effect of ICA on EEG quality: 

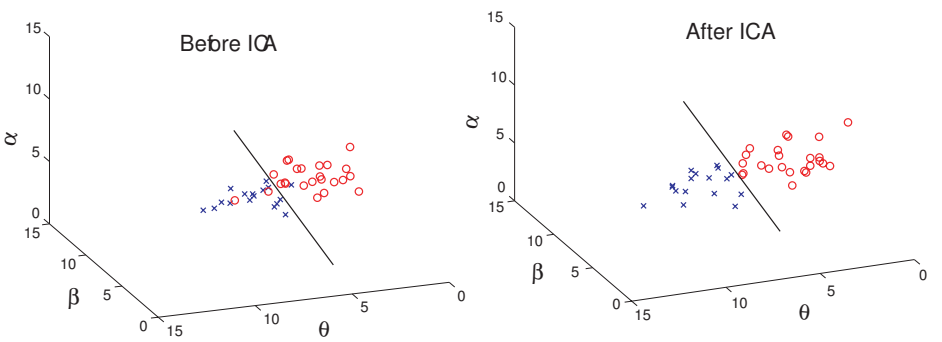

Fig. 3. Inter-subject variability of all subjects before and after ICA. Each control subject (circle) and mildAD patient (cross) are plotted a spatial representation of their Fourier power in the three lower frequency ranges - $\delta(1-4 \mathrm{~Hz}), \theta(4-8 \mathrm{~Hz})$ and $\alpha$ (8-12 Hz). Not only were subjects regrouped in their respective classes by ICA, but separation between the two groups was improved.

1. Fourier power distribution changed after ICA, the variability in at least one group increased (i.e., the variance of Fourier power increased in one group). In this case, ICA cleaning denatured EEG signals, as the data quality was lowered.

2. Fourier power distribution changed after ICA, the variability did not increase, and eventually decreased (i.e., the variance of Fourier power remained stable in both groups or decreased). In this case, ICA cleaning improved the quality of EEG signals.

We found the second situation, as a variance decrease in the control group was the only significant effect. We can therefore conclude that ICA cleaning does not denature EEG signals, and instead improves their quality. This results was however obtained with a combined cleaning done by three independant persons. Without such combination, human error might compensate with the benefits of $\mathrm{ICA}$ (error rate $\simeq 13.8 \%$ ). Other ways to combine cleaning obtained from several persons could be imagined. We also attempted to average spectrograms of each database: i.e. averaging each subject's three occurrences instead of selecting one occurrence. This led to very similar results, EEG data was not denatured. However, we believe that averaging does not prevent the intrusion of seriously flawed data, whereas our selection approach is more preemptive.

These results suggests a new direction for EEG studies using ICA: developping organized methodologies of cleaning, i.e. coordinated semi-automatic methods of EEG cleaning. In other words, instead of only developing new algorithms, we should endeavor to find markers of artifacts and semi-automatic methods of cleaning. An ideal toolbox should provide synthetic information about each ICA sources (indicating for instance a pre-diagnostic of anomalies); human cleaners would then perform a cleaning based on this information (a semi-automatic cleaning). 


\section{Acknowledgments}

J. Solé-Casals acknowledges support from the Ministerio de Educación y Ciencia of Spain (grant TEC2007-61535/TCM), and from the Universitat de Vic (grant R0912). Monique Maurice is supported by the JSPS fellowship P-08811.

\section{References}

1. Ille, N., Berg, P., Scherg, M.: Artifact correction of the ongoing eeg using spatial filters based on artifact and brain signal topographies. Journal of clinical neurophysiology 19 no.2 (2002) 113-124.

2. Jung, T.P., Makeig, S., Humphries, C., Lee, T.-W., McKeown, M.J., Iragui, V., Sejnowski, T.J.: Removing electroencephalographic artifacts by blind source separation. Psychophysiology 37 (2000) 163-178.

3. Cichocki, A., Blind Signal Processing Methods for Analyzing Multichannel Brain Signals. International Journal of Bioelectromagnetism, 6 no.1 (2004).

4. Cichocki, A., Shishkin, S.L., Musha, T., Leonowicz, Z., Asada, T., Kurachi, T.: EEG filtering based on blind source separation (BSS) for early detection of Alzheimer"s disease. Clinical Neurophysiology 116 no.3 (2006) 729-737

5. Anderer, P., Roberts, S., Schlogl, A., Gruber, G., Klosch, G., Herrmann, W., Rappelsberger, P., Filz, O., Barbanoj, M.J., Dorffner, G., Saletu., B.: Artifact processing in computerized analysis of sleep eeg - a review. Neuropsychobiology 40 no.3 (1999) $150-157$.

6. Van de Velde, M.: Signal Validation in Electroencephalography Research. PhD thesis, Technische Universiteit Eindhoven, Netherlands, January 2000.

7. Woon W.L., Cichocki A., Vialatte F., Musha T.: Techniques for early detection of Alzheimer's disease using spontaneous EEG recordings. Physiological Measurement 28 no. 4 (2007) 335-347.

8. Melissant, C., Ypma, A., Frietman, E.E., Stam, C.J.: A method for detection of Alzheimer's disease using ICA-enhanced EEG measurements. Artif Intell 33 no.3 (2005) 209-222.

9. Escudero, J., Hornero, R., Poza, J., Absolo, D., Fernndez, A.: Assessment of classification improvement in patients with Alzheimer's disease based on magnetoencephalogram blind source separation. Artif Intell Med 43 (2008) 75-85.

10. Cichocki, A., Amari, S., Siwek, K., Tanaka T., Phan A-H.: ICALAB version 3 toolbox, available online at http://www.bsp.brain.riken.jp/ICALAB/.

11. Henderson, G., Ifeachor, E., Hudson, N., Goh, C., Outram, N., Wimalaratna, S., Del Percio, C., Vecchio, F.: Development and assessment of methods for detecting dementia using the human electroencephalogram. IEEE Trans. Biom. Eng. 53 (2006) 1557-1568.

12. Barlow, J.S.: The Electroencephalogram: Its Patterns and Origins. Cambridge MA, MIT Press, 1993.

13. Tichavský, P., Yeredor, A., Nielsen, J.: A Fast Approximate Joint Diagonalization Algorithm Using a Criterion with a Block Diagonal Weight Matrix , Proc. ICASSP 2008, Las Vegas, U.S.A.

14. Yeredor, A.: Blind separation of Gaussian sources via second-order statistics with asymptotically optimal weighting. IEEE Signal Processing Letters 7 (2000) 197-200.

15. Tichavský, P., Doron, E., Yeredor, A., Nielsen, J.: A Computationally Affordable Implementation an Asymptotically Optimal BSS algorithm for AR Sources, EUSIPCO 2006, Florence, Italy. 